\title{
Aplastic Anemia-Related Mortality in Brazil, 2000-2018
}

\author{
Augusto Hasiak Santo* \\ Departamento de Epidemiologia, Faculdade de Saúde Pública da Universidade de São Paulo, São Paulo, \\ Brazil
}

*Corresponding author: Augusto Hasiak Santo, Departamento de Epidemiologia, Faculdade de Saúde Pública da Universidade de São Paulo, São Paulo, SP Brazil, 21 - Apt. 6201243-020, Tel: \#55-11-32584760; \#55-11-99019-3650

\begin{abstract}
Background: Nationwide population aplastic anemiarelated mortality is rare in the world medical literature.

Methods: The annual AA mortality data was extracted from the public databases of the Mortality Information System, searching category D61 "other aplastic anemias" of the International Classification of Diseases, Tenth Revision.

Main results: In Brazil, 2000 to 2018, 35,523 deaths related to AA occurred, $13.629(38.4 \%)$ as underlying cause, and $21,894(61.6 \%)$ as associated (non-underlying) cause of death. Average standardized mortality rates were for underlying, associated and all mentions cause deaths, respectively, $4.02,6.40$ and 10.42 deaths per million population. The overall mean and median ages at death for all mentions were, respectively, $51.5( \pm 24.7)$ and 55.5 (range 32.5-71.5) years. On certificates with AA as an associated cause, the underlying causes of deaths were neoplasms (38.9\%), infectious and parasitic diseases $(8.8 \%)$ of the circulatory system $(8.7 \%)$; the main underlying causes among the neoplasms were myeloid leukemia $(7.7 \%)$, lymphoid leukemia $(5.1 \%)$, other and specified types of non-Hodgkin's lymphoma (4.1\%), certain other neoplasm of lymphoid, hematopoietic and related tissue $(3.5 \%)$, and neoplasms of the digestive organs (3.2\%). The leading overall associated cause of death was septicemia, occurring in $48.9 \%$ among $\mathrm{AA}$ as the underlying cause and $42.6 \%$ among other underlying conditions, followed by pneumonias, respiratory failure, failure of multiple organs, renal failure, and cerebrovascular diseases.
\end{abstract}

Conclusion: This study revised AA death rate trends, underlying and associated causes of death, age at death and regional distribution of death in Brazil.

\section{Keywords}

Aplastic anemia, Mortality, Mortality trends, Cause of death

\section{Introduction}

Aplastic anemia (AA) is a rare and serious disease, potentially life-threatening, that affects hematopoietic stem and progenitor cells and is characterized by pancytopenia and a hypoplastic bone marrow. The development of AA result from the destruction of hematopoietic cells by three main mechanisms, including direct environmental injury, dysregulated immune system, and primary inherited or acquired bone marrow failure syndrome. There are no AA specific markers then the diagnosis is reached by exclusion of other rational entities. From 50 to 74 percent of AA cases of not have a clear cause and are labeled idiopathic. Patients may present signs and symptoms typical of cytopenia, such as infection and bleeding. The term "aplastic anemia" is somewhat a misleading terminology considering pancytopenia as its main characteristic. The most important treatments for AA are bone marrow transplantation and immunosuppressive treatment (IST) with antithymocyte globulin (ATC) and cyclosporine (CSA) [1-4].

Modern studies show an estimated incidence of 2-2.3 cases per million population per year in the Western countries [5]; a contemporary study in Sweden identified the incidence of 2.35 cases per million inhabitants per year [6]. The incidence in Asia is two to threefold higher, attaining 7.4 cases per million; a recent study disclosed an incidence of 5.67 cases per million population in Taiwan [7]. In Brazil, one pilot study disclosed the incidence of AA of 2.7 cases per million per year, ranging from 1.1 to 7.1 cases per million per year between regions [8], and, in the state of Parana,

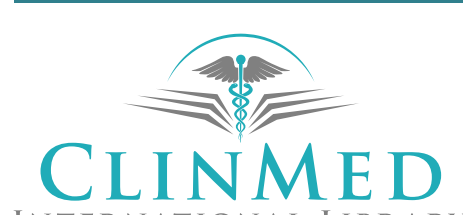

INTERNATIONAL LIBRARY
Citation: Santo AH (2022) Aplastic Anemia-Related Mortality in Brazil, 2000-2018. Int J Blood Res Disord 9:073. doi.org/10.23937/2469-5696/1410073

Accepted: January 12, 2022: Published: January 14, 2022

Copyright: (c) 2022 Santo AH. This is an open-access article distributed under the terms of the Creative Commons Attribution License, which permits unrestricted use, distribution, and reproduction in any medium, provided the original author and source are credited. 
a previous study from 1999 to 2000 found the annual incidence of AA 2.4 cases per million inhabitants [3]. In almost most studies, the sex ratio has been close of 1:1. Two patient age peaks of incidence are described, one among young adults and another in the elderly. In Parana, the age-specific incidence was bimodal, highest in those between 15 and 24 years old and there was another peak in patients 60 years or older [3]. The incidence of AA in Latin American countries was 1.6 cases per million per year [9].

The Brazilian national mortality statistics derive from death certificates that are supplied by doctors, or prepared through testimonies from witnesses, and are documented in Civil Registry Offices [10]. The demographic and medical data on death certificates are coded and processed by vital statistic or epidemiological surveillance services in the federal states and are sent to the Ministry of Health to be consolidated as data from the whole country [11]. Among these data, the value of causes of death is indisputable, of which the underlying cause is conceptualized as "(a) The disease or injury that initiated the train of morbid events leading directly to death, or (b) The circumstances of the accident or violence which produced the fatal injury" [12]. With the aim of preventing death, the primary mortality statistics are traditionally presented according to the underlying cause. Regardless of the great value of the underlying cause, across recent decades there has been increasing demand to consider all causes of death listed on death certificates, and not just the underlying cause. Such statistics are called "multiple-cause-of death", and they provide information on the whole range of fatal processes that culminate in death, thereby offering new elements and perspectives for their prevention [13]. This study aims to describe causes of death and mortality issues related to $A A$ based on death certificates data with the use of multiple-cause-of-death methodology.

\section{Methods}

Brazil, officially named Federative Republic of Brazil, is the world's fifth largest country, covering a total territory of 8.5 million $\mathrm{km}^{2}$, and the sixth largest population estimated in 212 million inhabitants in 2020. The country is politically and administratively divided into 27 federated units (26 states and the Federal District) and 5,570 municipalities. The 27 federated units are grouped into five geographic regions: North, Northeast, Southeast, South, and Central-West, The annual mortality data was extracted from the public multiplecause-of-death databases of the Mortality Information System (Sistema de informações sobre mortalidade - SIM) located at the Brazilian Unified Health System Information Technology Department (Departamento de informática do sistema único de saúde - DATASUS), ministry of health (MS) [14]. All deaths were selected in which AA as a cause of death was listed on any line or in either part of the International Form of Medical
Certificate of Cause-of-Death (the medical certification section of the death certificate), irrespective of whether characterized as the underlying cause-of-death or as an associated (non-underlying) cause. Complications of the underlying cause (Part I of the medical certification section) and contributing causes (Part II of the medical certification section) were jointly designated as associated (non-underlying) causes of death [13]. We employed the 2000-2018 mid-year estimates of the population for Brazil, discriminated by year, sex, age group and Brazilian regions.

According to the International Classification of Diseases and Related Health Problems, Tenth Revision (ICD-10), AA as a cause is described in category D61 - Other Aplastic anemias, including four character subcategories codes D61.0 Constitutional Aplastic anemia, D61.1 Drug-induced aplastic anemia, D61.2 Aplastic anemia due to other external agents, D61.3 Idiopathic aplastic anemia, D61.8 Other specified Aplastic anemia, and D61.9 Aplastic anemia, unspecified [15].

To reconstruct the morbid process leading to death, all causes of death listed in the medical certification section of the death certificate were considered, including those classified as ill-defined, equated as such, or considered by the world health organization (WHO) as modes of death $[12,15]$.

Records included in the mortality databases contain fields like the ones appearing on official Brazilian death certificate. In addition, we created auxiliary fields for the study of multiple causes, including a field designed to contain a single "string" of characters composed of the codes entered on lines (a), (b), (c) and (d) of Parts I and of Part II of the medical certification section of the death certificate.

The causes of death had been automatically processed with the software Underlying Cause Selector (Seletor de causa básica SCB) [16]. The automatic processing involves the use of algorithms and decision tables that incorporate the WHO mortality standards and the etiological relationships among the causes of death. The expressions "death from" and "death due to" refer to the underlying cause-of-death, whereas "deaths with a mention of" and "mortality related to" refer to the listing of a given condition either as the underlying cause or as an associated (non-underlying) cause. The causes of death evaluated in the present study were those mentioned in the medical certification section, which are known internationally as "entity axis codes", defined and presented under the structure and headings of the ICD [13].

Using mortality rates, proportions, and historical trends, we studied the distributions of the following variables: Sex, age at death (in ten year age-groups), year of death, underlying cause-of-death, associated (non- 
underlying) cause(s) of death, total mentions of each cause-of-death, mean number of causes listed per death certificate, and geographical distribution of deaths. Medical and demographic variables were processed with the following software: dBASE III Plus, version 1.1 and dBASE IV (Ashton-Tate Corporation, Torrance, CA), Epi Info, version 6.04d (Centers for Disease Control and Prevention, Atlanta, GA), in emulated $\mathrm{dbDOS}^{\mathrm{TM}}$ PRO 6 environment, Excel 2016 (Microsoft Corporation, Redmond, WA). We used the Multiple Causes Tabulator (Tabulador de Causas Múltiplas for Windows) program (DATASUS, Ministério da Saúde, Faculdade de Saúde Pública, Universidade de São Paulo, Brazil), to process ICD-10 codes (TCMWIN, version 1.6), in our presentation of the associated causes and of the mean number of causes per death certificate [17].

For the presentation of the associated (nonunderlying) causes listed on the death certificates on which AA was identified as the underlying cause, special lists were prepared showing the causes involved in the respective natural histories [1,2], as well as those mentioned with the greatest frequency. The duplication or multiplication of causes of death was avoided when these were presented in abbreviated lists. The number of causes depends on the breadth of the class (subcategory, category, grouping or chapter of the ICD10); therefore, if two or more causes mentioned in the medical certification section were included in the same class, only one cause was computed $[16,17]$.

Mortality rates (per $1,000,000$ population) for AA were calculated-by year and for the study period (20002018) as a whole-based on the number of deaths that had been identified as the underlying cause or as an associated (non-underlying) cause, as well as on the overall number of its mentions. To calculate the average mortality rate, the overall number of deaths was divided by the sum of the respective annual population counts for the 19-year study period.

The Programa para Análisis Epidemiológico de Datos (Epidat, Epidemiological Analysis of Data Program), version 4.2 (Dirección Xeral de Innovación e Xestión da Saúde Pública, Xunta de Galicia, and Pan American Health Organization) was used for standardize, by the direct method, the sex and age-adjusted crude and average mortality rates for the study period as a whole, to the new WHO Standard Population [18].

We used analysis of variance to compare the mean numbers of causes mentioned on the death certificate and the Kruskal-Wallis $\mathrm{H}$ test to compare the median age at death between groups. The Join point Regression Program, version 4.8.0.1, was used to evaluate the changes in age-standardized rates trends [19]. Assuming a Poisson distribution, join point analysis chooses the best fitting point (or points), at which the rate increases or decrease significantly. Values of $p<0.05$ were considered significant.

\section{Results}

During the period of 19 years, from 2000 to 2018, in Brazil 35,523 deaths related to aplastic anemia occurred, $13.629(38.4 \%)$ as the underlying cause and $21,894(61.6 \%)$ as an associated (non-underlying) cause of death. As the underlying cause, Aplastic anemia varied from $31.9 \%$ in 2005 to $45.5 \%$ in 2018 , from $30.1 \%$ at $30-39$ years of age to $53.5 \%$ at 80 and older deaths, and from $36.9 \%$ in the Center West region to $44.3 \%$ in the North region. Overall, males accounted for $51.7 \%$ and females $48.3 \%$ of deaths; whereas $50.2 \%$ in the Southeast, $21.6 \%$ in the Northeast, $15.3 \%$ in the South, $7.2 \%$ in the Center West, and $5.6 \%$ in the North regions. The ICD-10 subcategory D61.9. Aplastic anemia, unspecified, was used $90.0 \%$ and $94.6 \%$ to identify, respectively, AA as the underlying and as an associated (non-underlying) cause of death (Table 1).

Mortality rates for the period 2000-2018 are presented in Table 2 and Figure 1. Average standardized mortality rates for underlying, associated (nonunderlying) and all mentions causes deaths were, respectively, 4.02, 6.40 and 10.42 deaths per million. Underlying causes deaths increased from 2000 to 2013 by annual percent change (APC) of $1.41 \%$, followed by a decrease up to 2018 (APC -3.29\%); associated (nonunderlying) causes deaths increased from 2000 to 2002 (APC 9.76), followed by a drop to 2018 (APC -3.14), and all mentions causes death increased from 2000 to 2002 (APC 6.11) followed declines to 2013 (APC -1.7) and to 2018 (APC -4.1). These observed trends resulted in average annual percent changes (AAPC) for underlying, associated (non-underlying) and all mentions deaths respectively of $0.1,-1.8$ and -1.2 for the whole period. Averaged age-specific death rates stayed leveled until ages 40 to 49 when increased exponentially thereafter, reaching, at the age of 80 and older, the rates of $44,11,38.41$ and 82.51 deaths per million population, respectively for underlying, associated (non-underlying) and all mentions causes (Figure 2).

Mean and median ages at death related to aplastic anemia are presented on Table 3. Overall general mean and median ages at death for all mentions deaths were, respectively, 51.5 ( \pm 24.7$)$ and 55.5 (32.5-71.5) years. Deaths at older mean and median ages occurred with underlying causes, in females, in the South region and at home, while deaths at earlier ages were observed with associated (non-underlying) causes, in males, in the North region, and as underlying cause in public thoroughfare. Ages at death in females were always older than in males.

The underlying causes on 35.519 death certificates for male and female in which AA was listed as a cause of death are presented in Table 4, according to the ICD structure. Overall, diseases of blood and blood forming organs are the leading underling cause, totaling $40.1 \%$, 


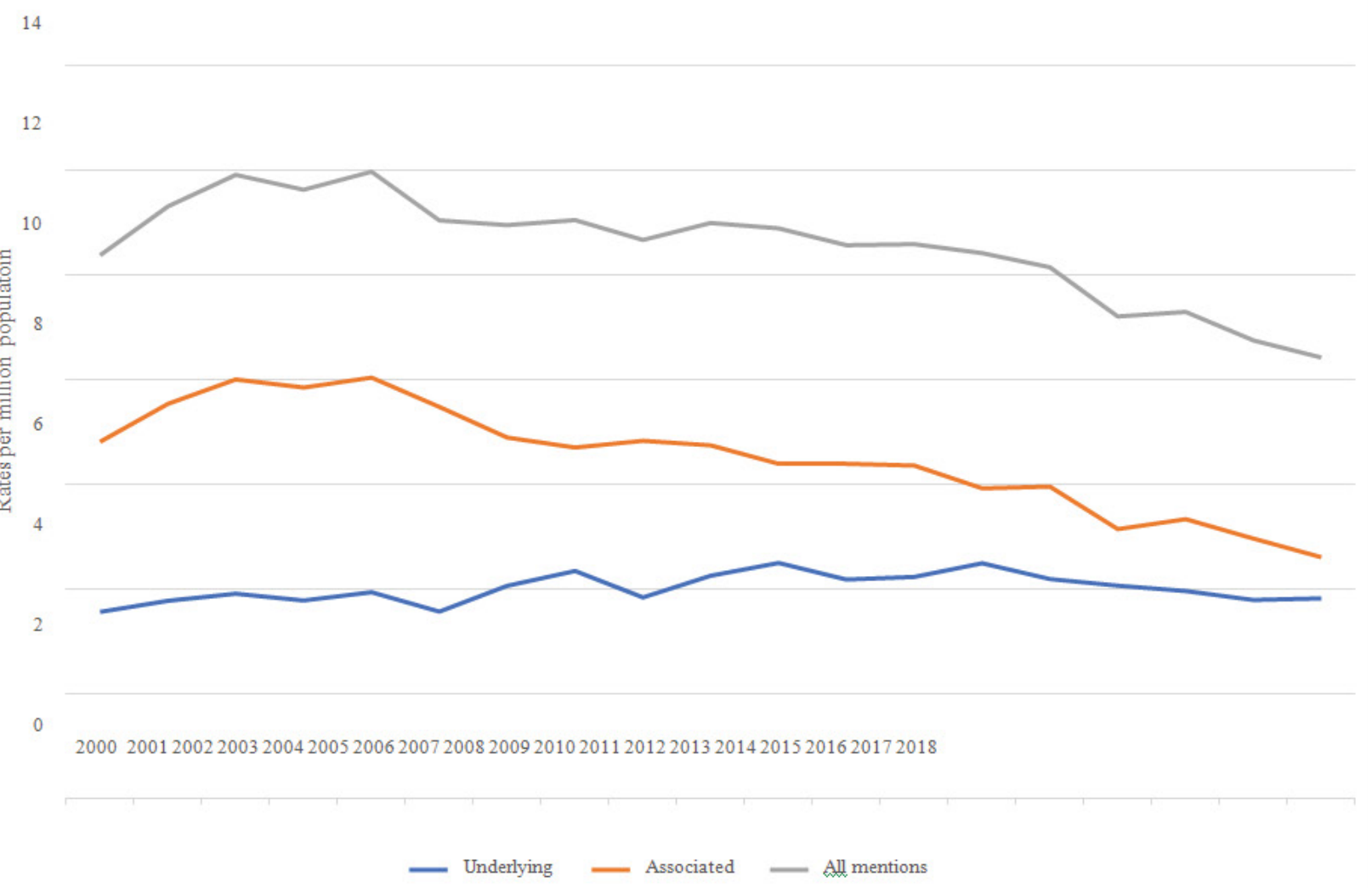

Figure 1: Age-standardized death rates related to aplastic anemia according to causes of death in Brazil, 2000 to 2018.

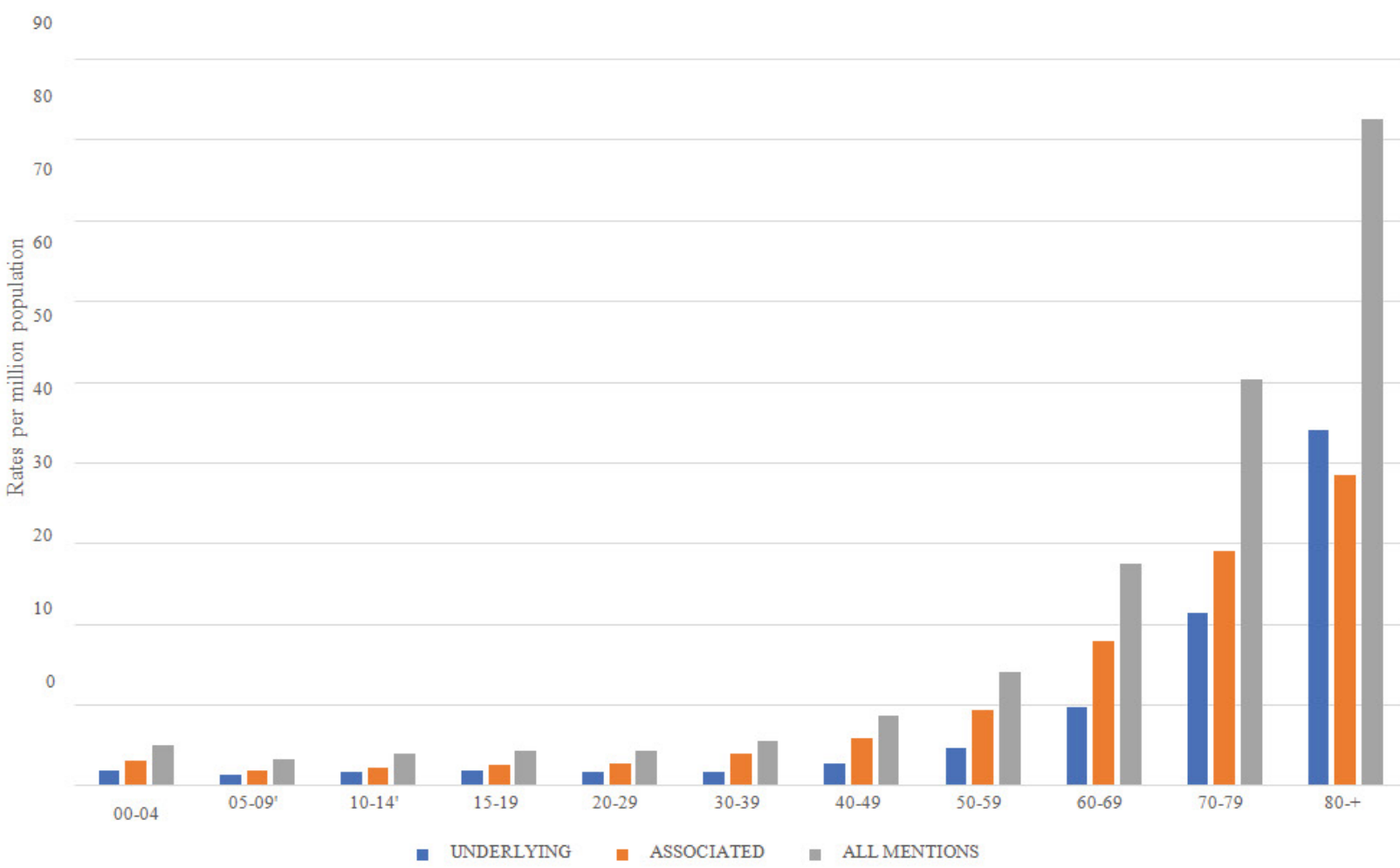

Figure 2: Specific age death rates related to aplastic anemia according to causes of death, Brazil, 2000 to 2018.

with AA itself reported for $38.4 \%$ of deaths. Next and remarkably close, the ICD chapter on neoplasms emerges, totaling $38.9 \%$, followed by infectious and parasitic diseases, with $8.8 \%$ of all deaths. The main underlying causes among the neoplasms were myeloid leukemia (7.7\%), lymphoid leukemia (5.1\%), other and specified types of non-Hodgkin's lymphoma (4.1\%), certain other neoplasm of lymphoid, hematopoietic and related tissue (3.5\%), and neoplasms of the digestive organs (3.2\%). Human immunodeficiency virus disease 
prevailed among the infectious diseases accounting overall for $5.8 \%$ of deaths, in males $7.3 \%$, and in females $4.2 \%$.

The leading associated (non-underlying) causes of deaths listed on death certificates which AA and other selected conditions were identified as the underlying causes are displayed in Table 5, according to the ICD structure. The overall associated (non-underlying) causes included in chapters on infectious, respiratory, and ill-defined conditions stand out holding the percentages respectively of $45.0 \%, 37.1 \%$ and $35.6 \%$. Conclusively, the septicemias, occurring in $48.9 \%$ among $\mathrm{AA}$ as the underlying cause and $42.6 \%$ among other underlying conditions, occurs as the major associated (non-underlying) cause, followed overall by pneumonias, respiratory failure, failure of multiple organs, renal failure, and cerebrovascular diseases.

\section{Discussion}

To our knowledge, following extensive pursue on Pub Med and Embase, after thirty years, this study is a renewed research on $A A$ nationwide population mortalitystatistics [20]. This study describes the mortality related to $A A$ in Brazil in the last 19 years considering all its mentions as cause of death on death certificates. Usually, the account of incidence studies removes cases of neoplastic or granulomatous disease involving the associated (non-underlying) with pancytopenia, such as myelodysplastic syndrome, Fanconi's anemia, and paroxysmal nocturnal hemoglobinuria, as well patients exposed to antineoplastic chemotherapy or radiotherapy. In contrast, the benefits of the present multiple cause of death approach contemplate all death certificates in the whole country where AA was listed as the underlying cause, as well as an associated (nonunderlying) cause of death, providing a more complete estimate of nationwide mortality.

The ICD-10 subcategory aplastic anemia, unspecified (D61.9) was used over ninety percent in the classification of $\mathrm{AA}$ as underlying and associated (non-underlying) causes of death. A clear etiologic factor has not been identified and listed on the death certificate [1]. The misleading and confusing terminology "aplastic anemia" is another related issue considering pancytopenia, with decreased platelets, with cells, and erythrocytes, as the main characteristic of the condition. Such a bone marrow failure of this nature should be called "Aplastic pancytopenia" [1,21]. Perhaps this proposal might be placed in an international congress of hematology.

The finding that AA was identified on average as the underlying cause in $38.4 \%$ of the death certificates does not reflect the severity of this condition in Brazil. This value is even lower than $55 \%$ listed as underlying cause among AA related deaths in the United States in 1984 [20]. A latest review describes the fatality rate of $85 \%$ for severe AA [22]. Traditionally, higher percentages of a given cause of death as the underlying cause is like a statement of its severity. Assaults, transport accidents, suicides, drownings, and certain neoplasms sites are common such causes in Brazil selected over $95 \%$ as the underlying cause of death [23]. A possible reason for the lower AA percent as underlying cause could stay in the constellation of conditions that delineate the pathophysiology of $A A$, including myelodysplastic syndrome, lymphoid, hematopoietic, and related tissue malignant neoplasms of severe fatality rates.

The observed AA averaged standardized overall mortality rate of 10.42 deaths per million population is equivalent to the estimated crude death rate of 8.9 deaths per million population verified in the United States in 1984, as well as the corresponding underlying cause of death rates of 4.02 and 4.90 per million inhabitants, respectively in Brazil and United States [20]. Globally, the AAPC indicated that underlying cause of death mortality trends stayed leveled the whole study period, while associated (non-underlying) cause of death rate trends revealed a regular decrease from 2002 to 2018. Averaged standardized death rates arise exponentially from 50 years of age and reach the highest levels in the oldest fatalities. This pattern has been observed in other studies. In the United States research it has been speculated that the high level of exposure among older age persons to medicinal agents and the high susceptibility of the aged to drug-associated blood dyscrasi as might contribute to these high death rates [20].

The median age at death for overall all mentions AA deaths of 55.5 years was much lower than 76 years of age found in the United States in 1984. The male to female ratio was equivalent to $1: 1$ observed in incidence studies.

Among overall death certificates in Brazil which included a mention of AA as a cause of death, the major underlying causes emphasized diseases of blood and blood forming organs, AA prevailing, and malignant neoplasms, mostly myeloid and lymphoid leukemias, both causes mutually accounting for $79 \%$ of all deaths. The underestimation of AA severity as underlying cause has been noticed earlier. Studies have shown that $A A$ survivors patients may harbor high risk of malignant neoplasms, owing a possible association with AA itself as well as following immunosuppressive treatment or bone marrow transplantation [24]. Myelodysplastic syndromes, acute leukemias and solid neoplasm ate the mostcommonassociatedcancers.Studieshavesuggested that survivors of $A A$ that received immunosuppressive therapy are susceptible to hematologic cancers while patients after bone marrow transplantation present increased incidence of solid tumors [25]. Regrettably, the use solely of death certificate data does not allow the recovery of the evolution of an aplastic anemia to subsequent hematopoietic or solid neoplasms or vice- 
versa. Another constraint relates to the unavailability to compare the observed underlying causes of death with other countries or studies; the search for papers on the terms "aplastic anemia" and "underlying cause of death" in Pub Med and Embase produce only one paper on mortality from intractable diseases in Japan from 1972 to 2004.

This study has confirmed infectious diseases as a leading associated (non-underlying) cause of death, accounting $48.9 \%$ and $42.6 \%$, respectively, among AA underlying and non-underlying deaths, where septicemias prevail, tallying above $47 \%$ overall deaths. Incidentally, infections are defined as severe when evidence of sepsis or septic shock occurs [25]. Additionally, the onus of pneumonias associated mainly among AA underlying cause deaths was verified. The major infectious associated mortality is due to the universal presence of severe and prolonged neutropenia in AA patients with increased susceptibility to infections caused by bacteria and fungi. A study in AA children in Italy found a significant association between the grade of aplasia at diagnosis and incidence of infection episodes, stressing the severity of $A A$ as the most important factor for the development of an infection [26]. Most infections also occur after patients received IST, as a combination of adrenal corticosteroids, cyclosporin and antithymocyte globulin (ATG) $[24,26,27]$. In the United States, a study found that older age was an independent factor associated with 1-year mortality in multivariate analysis of non-respondent IST patients [28]. In France, among elderly AA patients, mainly infections were the major complications almost exclusively of IST, especially anti-thymocyte globulin (ATG) associated with cyclosporine-A (ATG-CsA) [29].

Population mortality statistics suffer from quantity and quality problems, while some specific issues related to $A A$ were discussed in previous paragraphs. We intend to emphasize the advantages and contribute to overcome the limitations of death certificates mortality data. Estimations for 2017 indicate a coverage of $96.3 \%$ for the whole country, ranging from $92.7 \%$ in the North region to $100.0 \%$ in the South region [30]. Regarding quality, recent evaluation of the institution of multiple cause of death statistics in Brazil, from 2003 to 2015, has found that the crude mean number of causes per death certificate increased from 2.81 to 3.02 (7.5\%), deaths with only one mentioned cause decreased from $20.32 \%$ to $13.75 \%$ and ill-defined causes of death as underlying cause decreased from $12.95 \%$ to $5.59 \%$ $(56.22 \%)[31,32]$. Particularly in this study, the mean number of causes per death certificates were 3.54 ( \pm $1.26)$ and 4.33 ( \pm 1.21 ), respectively, on AA underlying and associated (non-underlying) causes deaths.

However, a validation study performed in La Rioja, Spain, during the period 2007-2012, confirmed only $15 \%$ of true AA diagnosis included in hospital database or mortality registry, even though removing patients and deaths associated to the AA related conditions like in customary incidence studies [33]. Though automatic processing of mortality data is used in Brazil, causes of death are still done by trained nosologists who might make a mistake and introduce a wrong ICD code. Another question is linked with $A A$ as a rare cause of death while decision tables for automatic processing may not include ICD mortality rules and dispositions involving all conditions of its natural history. And finally, most important to remember the responsibility of physicians to correctly identify causes of death on death certificate.

\section{Conclusions}

Data on AA nationwide causes of death and mortality issues in Brazil from 2000 to 2018 were presented. Someway unexpected, even though the main underlying cause, AA was thus identified below half of deaths, followed closely by hematopoietic, lymphoid, and solid malignant neoplasms, causes whose figures ought to have detailed research. Septicemias and pneumonias as the overall leading associated (non-underlying) cause of death reiterated the severity of $A A$ neutropenia. However, despite the stated results, further questions remain about $A A$ concerns hat deserve attention, such as the accurate identification of $A A$ as true cause of death, the occurrence of subsequent neoplasms after IST or HCBT and the association of AA and malignant hematopoietic neoplasms.

\section{References}

1. Young NS (2018) Aplastic anemia. N Engl J Med 379: 1643-1656.

2. Furlong E, Carter T (2020) Aplastisc anaemia: Current concepts in diagnosis and management. Journal of Paediatrics and Child Health. Blackwell Publishing 56: 1023-1028.

3. Maluf EMCP, Pasquini R, Eluf JN, Kelly J, Kaufman DW (2002) Aplastic anemia in Brazil: Incidence and risk factors. Am J Hematol 71: 268-274.

4. Clucas DB, Fox LC, Wood EM, Hong FS, Gibson J, et al. (2019) Revisiting acquired aplastic anaemia: Current concepts in diagnosis and management. Intern Med J 49: 152-159.

5. Young NS, Kaufman DW (2008) The epidemiology of acquired aplastic anemia. Haematologica 93: 489-492.

6. Vaht K, Goransson M, Carlson K, Isaksson C, Lenhoff S, et al. (2017) Incidence and outcome of acquired aplastic anemia: Real-world data from patients diagnosed in Sweden from 2000-2011. Haematologica 102: 1683-1690.

7. Li SS, Hsu YT, Chang C, Lee SC, Yen CC, et al. (2019) Incidence and treatment outcome of aplastic anemia in Taiwan-real-world data from single- institute experience and a nationwide population-based database. Ann Hematol 98: 29-39.

8. Hamerschlak N, Maluf E, Pasquini R, Eluf-Neto J, Moreira $\mathrm{FR}$, et al. (2005) Incidence of aplastic anemia and agranulocytosis in Latin America - The Latin Study. Sao 
Paulo Med J 123: 101-104.

9. Maluf E, Hamerschlak N, Cavalcanti AB, Avezum A, ElufNeto J, et al. (2000) Incidence and risk factors of aplastic anemia in Latin American countries: The LATIN casecontrol study. Haematologica 94: 1220-1226.

10. (1973) Dispoe sobre os registros publicos, e da outras providEncias. DiArio Oficial da Uniao.

11. (1999) Ministerio da Saude. Sistema de informacoes sobre mortalidade: Manual de procedimentos. Brasilia: Ministerio da Saude.

12. World Health Organization (2011) International statistical classification of diseases and related health problems. Tenth Revision. Vol. 2. Instruction manual. World Health Organization, Geneva.

13. Santo AH (1989) Causas multiplas de morte: Formas de apresentacao e metodos de analise.

14. Ministerio da Saude. Departamento de Informatica do SUS. Acesso a Informacao. Servicos. transferencia-downloadde-arquivos. arquivos-de-dados.

15. World Health Organization (1993) International statistical classification of diseases and related health problems. Tenth Revision. Vol. 1. World Health Organization, Geneva.

16. Santo AH, Pinheiro CE (1995) Uso do microcomputador na selecao da causa basica de morte. Bol Oficina Sanit Panam 119: 319-327.

17. Santo AH, Pinheiro CE (1999) Tabulador de causas multiplas de morte. Rev Bras Epidemiol 2: 90-97.

18. Ahmad OB, Boschi-Pinto C, Lopez AD, Murray CJL, Lozano $R$, et al. (2001) Age standardization of rates: A New WHO Standard.

19. (2019) Joinpoint regression program, Version 4.7.0.0. Statistical research and applications branch, national cancer institute.

20. Hine LK, Burt Gerstman B, Wise RP, Tsong Y (1990) Mortality resulting from blood dyscrasias in the United States, 1984. Am J Med 88: 151-153.

21. Cuglievan B, DePombo A, De Angulo G (2016) Aplastic anemia: The correct nomenclature matters. Haematologica.

22. Bassani BFB, Schuster AL, Chaves JSI, Crippa CM, Dambros VL, et al. (2020) Anemia aplastica: Definicao e tratamento. Hematol Transfus Cell Ther 42: 2-3.
23. Santo AH (2007) Potencial epidemiologico da utilizacao das causas multiplas de morte por meio de suas mencoes nas declaracoes de obito, Brasil, 2003. Rev Panam Salud Publica/Pan Am J Public Health 22: 178-186.

24. Socie G, Henry-Amar M, Bacigalupo A, Hows J, Tichelli A, et al. (1993) Malignant tumors occurring after treatment of aplastic anemia. N Engl J Med 329: 1152-1157.

25. Torres HA, Bodey GP, Rolston KVI, Kantarjian HM, Raad II, et al. (2003) Infections in patients with aplastic anemia: Experience at a tertiary care cancer center. Cancer 98: 8693.

26. Quarello P, Saracco P, Giacchino M, Caselli D, Caviglia I, et al. (2012) Epidemiology of infections in children with acquired aplastic anaemia: $A$ retrospective multicenter study in Italy. Eur J Haematol 88: 526-534.

27. Valdez JM, Scheinberg P, Young NS, Walsh TJ (2009) Infections in patients with aplastic anemia. Semin Hematol 46: $269-276$.

28. Valdez JM, Scheinberg P, Nunez O, Wu CO, Young NS, et al. (2011) Decrease infection-related mortality and improved survival in severe aplastic anemia in the past two decades. Clin Infect Dis 52: 726-735.

29. Contejean A, Resche-Rigon M, Tamburini J, Alcantara M, Jardin F, et al. (2019) Aplastic anemia in the elderly: A nationwide survey on behalf of the french reference center for aplastic anemia. Haematologica 104: 256-262.

30. (2020) Indicadores de cobertura que utilizam a metodologia do Busca Ativa - Indicadores - Busca Ativa - Indicadores, Acoes e Programas - Acesso a Informacao - DASNT - SVS/ MS.

31. Santo AH, Pinheiro CE (2015) Reavaliacao do potencial epidemiologico das causas multiplas de morte no Brasil, 2015. Research Gate, preprint.

32. Santo AH, Pinheiro CE (2019) Reassessment of the epidemiological multiple-cause-of- death potential use in Brazil, 2015. In: Presented at the fourth international meeting on multiple cause-of-death analysis, Institut national d'etudes demographiques, INED.

33. Ruiz E, Ramalle-Gomara E, Quinones C, Rabasa P, Pison C (2015) Validation of diagnosis of aplastic anaemia in La Rioja (Spain) by International Classification of Diseases codes for case ascertainment for the Spanish National Rare Diseases Registry. Eur J Haematol 94: 400-403. 